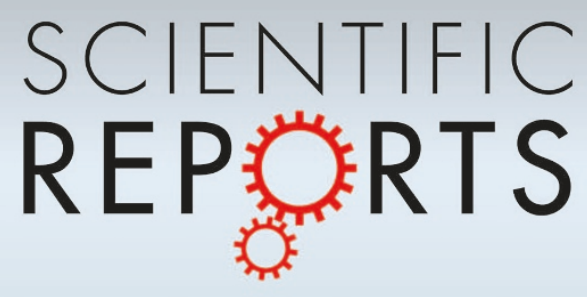

OPEN

SUBJECT AREAS:

MATERIALS SCIENCE

MATERIALS FOR ENERGY AND

CATALYSIS

PHYSICS

X-RAYS

Received

29 November 2012

Accepted

14 February 2013

Published

14 March 2013

Correspondence and requests for materials should be addressed to

J.S.R. (ross36@llnl. gov)

\section{Lead (Pb) Hohlraum: Target for Inertial Fusion Energy}

\author{
J. S. Ross', P. Amendt', L. J. Atherton', M. Dunne', S. H. Glenzer', J. D. Lindl', D. Meeker', E. I. Moses', \\ A. Nikroo ${ }^{2} \&$ R. Wallace' \\ 'Lawrence Livermore National Laboratory, P. O. Box 808, Livermore, CA 94551, USA, ${ }^{2}$ General Atomics, San Diego, CA 92121 , \\ USA.
}

Recent progress towards demonstrating inertial confinement fusion (ICF) ignition at the National Ignition Facility (NIF) has sparked wide interest in Laser Inertial Fusion Energy (LIFE) for carbon-free large-scale power generation. A LIFE-based fleet of power plants promises clean energy generation with no greenhouse gas emissions and a virtually limitless, widely available thermonuclear fuel source. For the LIFE concept to be viable, target costs must be minimized while the target material efficiency or $\mathrm{x}$-ray albedo is optimized. Current ICF targets on the NIF utilize a gold or depleted uranium cylindrical radiation cavity (hohlraum) with a plastic capsule at the center that contains the deuterium and tritium fuel. Here we show a direct comparison of gold and lead hohlraums in efficiently ablating deuterium-filled plastic capsules with soft $\mathrm{x}$ rays. We report on lead hohlraum performance that is indistinguishable from gold, yet costing only a small fraction.

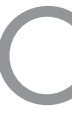
urrent LIFE designs ${ }^{1,2}$ focus on lead $(\mathrm{Pb})$ as the primary hohlraum material due to its low cost, low melting point and compatibility with the recycling requirements for minimized material use. A schematic of the power cycle envisioned for a LIFE power plant is shown in Figure 1. With a shot rate of $\sim 16 \mathrm{~Hz}$ a LIFE power plant will require over five hundred million targets per year. The price of lead is approximately one dollar per pound; a similar amount of gold would cost over 20,000 times that amount, and would present insurmountable technical obstacles for the LIFE target chamber due to its comparatively high melting point. Thus, the continued use of gold as a target material is incompatible with application to inertial fusion energy (IFE). However, gold has been the traditional hohlraum material of choice due to its fabrication ease, high $\mathrm{x}$-ray conversion efficiency, and the modestly low quantities required in experiments. The use of $\mathrm{Pb}$ in inertialconfinement-fusion (ICF) hohlraum experiments has been seldom considered despite its relative abundance, owing to concerns over oxidation and a predicted higher wall energy loss (from 3\% to 15\% depending on the model and alloy used $)^{2-4}$. A summary of the projected hohlraum material costs for an IFE power plant is shown as an inset in Figure 1. For ongoing research efforts in ICF, hohlraum fabrication costs are presently substantial, but for an IFE power plant an economy of scale using $\mathrm{Pb}$ is calculated to reduce fabrication costs to nearly 30 cents per target $^{5}$. What remains however is to demonstrate that $\mathrm{Pb}$ is an acceptable hohlraum material from a physics performance standpoint.
\end{abstract}

\section{Results}

A series of experiments has begun to explore candidate target materials such as $\mathrm{Pb}$, initially using the OMEGA laser facility ${ }^{6}$ and reduced-scale targets to reproduce ICF-relevant hohlraum drive temperatures. The target geometry is shown in Figure 2 (a). The target is a cylindrical vacuum lead hohlraum $2.3 \mathrm{~mm}$ in length and $1.6 \mathrm{~mm}$ in diameter with a pair of $0.8 \mathrm{~mm}$ diameter laser entrance holes. The hohlraum is heated with $14 \mathrm{~kJ}$ of $3 \omega 351 \mathrm{~nm}$ wavelength Nd:glass laser energy delivered by 38 beams, which heat the hohlraum wall, in turn radiating $x$ rays that ablate the capsule and compress the fuel. In a LIFE power plant the hohlraum will provide the soft $\mathrm{x}$-ray radiation drive and additionally protect the fuel capsule from debris and background radiation during transit of the target chamber. After ignition, the hohlraum also serves to protect the vacuum chamber walls from high energy ions produced in the fusion reaction. On OMEGA a diagnostic window with a diameter of $0.4 \mathrm{~mm}$ is located $0.2 \mathrm{~mm}$ away from the hohlraum equator and is used to image $\mathrm{x}$-ray self-emission from the imploding capsules. The plastic $(\mathrm{CH})$ capsules have an inner diameter of $460 \mu \mathrm{m}$, a wall thickness of $24 \mu \mathrm{m}$, and are suspended by a Formvar ${ }^{\circledR}$ web for placement at the center of the hohlraum. Twenty atmospheres of deuterium gas are used to fill the capsule and 0.025 atm of argon is added to enhance $3-5 \mathrm{keV}$ x-ray core imaging. 


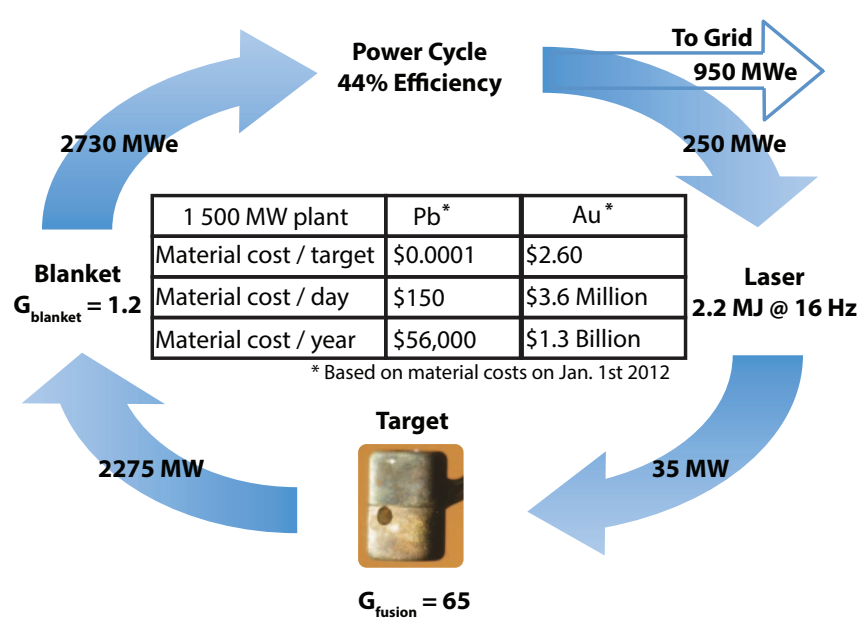

Figure 1 The power cycle of a LIFE power plant. Inset is a cost comparison between $\mathrm{Au}$ and $\mathrm{Pb}$ hohlraums assuming 16 target shots per second.

Figure 2 (b) shows an example of an x-ray image of the symmetrically compressed capsule core. Symmetric compression is critical for robust ICF performance and is typically parameterized as a decomposition of Legendre polynomials $\mathrm{P}_{\mathrm{n}}$. Low order even modes $\mathrm{s}^{7,8}$ result from intrinsic asymmetries in the soft $x$-ray drive. For the $30 \%$ contour shown in Figure 2 (b), $\mathrm{P}_{0}=61 \pm 3 \mu \mathrm{m}$, which represents about a factor-of- 4 compression from the initial capsule inner radii of $230 \pm 10 \mu \mathrm{m}$. Experiments on the National Ignition Facility (NIF $)^{10}$ and an eventual LIFE power plant will require $\sim 30$-fold compression. Here, we observed excellent symmetry and compression with $\mathrm{P}_{2}$ $=2 \pm 1 \mu \mathrm{m}$, and $\mathrm{P}_{4}=2 \pm 1 \mu \mathrm{m}$, consistent with the expected requirements for ICF implosions ${ }^{11}$, albeit at a less challenging convergence ratio.

These implosions have been carried out using two temporally shaped laser pulses, as shown in Figure 3 (a). The initial 1 ns of the pulse or "foot" is varied between pulse shapes to span the effective $1^{\text {st }}$ shock pressure delivered to the capsule for several ignition ablator candidates, including $\mathrm{CH}, \mathrm{Be}$ and high-density carbon. The low foot pulse shape results in a pressure of $\sim 3.6 \mathrm{Mbar}$ and the $2 \times$ foot pulse in a pressure of $\sim 7.5$ Mbar during the foot of the laser pulse. These pressures are very close to one and two times the pressure at the center of the earth, $3.6 \mathrm{Mb}^{13}$, and could be used as a platform for studies of planetary physics in the laboratory ${ }^{14}$. The length of the pulse is then adjusted based on simulations to optimize the shock timing in the capsule shell. Multiple shocks are used to efficiently compress the capsule and fuel. Fast, strong shocks are more stable, but they increase the entropy of the target and make it harder to
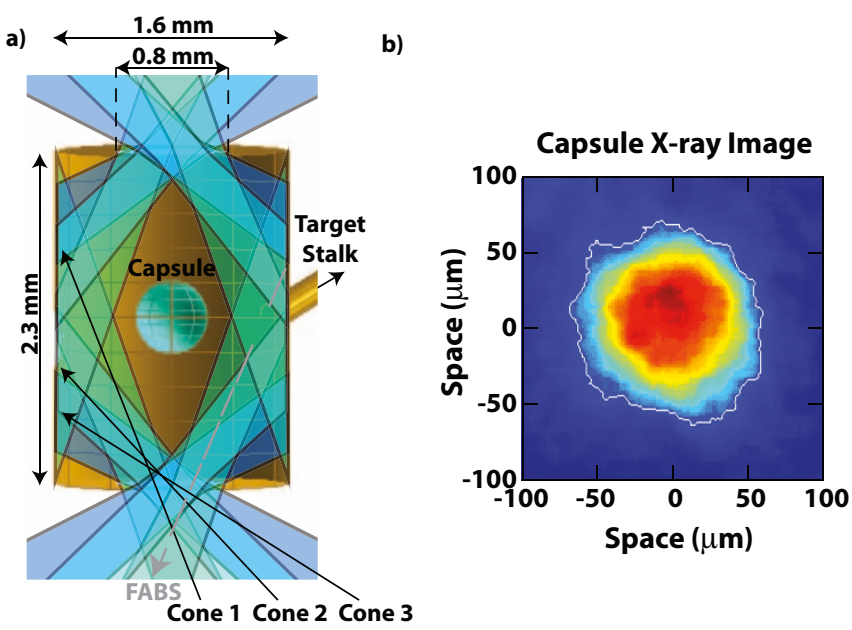

Figure $2 \mid$ (a) The hohlraum target geometry is shown. 38 laser beams enter the hohlraum through 2 laser entrance holes with diameters of $0.8 \mathrm{~mm}$. (b) An x-ray self-emission image ${ }^{9}$ of the compressed capsule.

compress. The goal with shock timing in ICF is to find the correct balance.

The laser energy delivered to the hohlraum is converted to $\mathrm{x}$ rays and characterized through the laser entrance hole using a calibrated array of $\mathrm{x}$-ray diodes (DANTE) ${ }^{15}$. The backscattered laser energy is characterized using the full-aperture backscatter station (FABS) and was measured to be less than $2 \%$ for both hohlraum materials for the cone 1 laser beams. The measured radiation drive is compared for each material in Figure 3 (b). In contrast to the expectations from the earlier work ${ }^{2,3}$, the gold and lead hohlraums produce similar peak radiation temperatures of $225 \mathrm{eV}$, within the uncertainty $( \pm 5 \mathrm{eV})$ of the diagnostic for both laser pulse shapes. The peak temperature is sufficiently close to the requirement of $\sim 250 \mathrm{eV}^{2}$ planned for a LIFE power plant that these experiments provide representative data.

Importantly, the measured high performance of the $\mathrm{Pb}$ hohlraums occurred even in the presence of oxidation and thus surface degradation of the hohlraum, in contrast to the mirror-like finish of the gold hohlraum. The oxidation was bounded using Auger spectroscopy at less than 5 at.\% O after $\sim 50 \mathrm{~nm}$ into the interior hohlraum surface (See Supplementary Section S.1).

\section{Discussion}

The performance of the deuterium-filled $\mathrm{CH}$ capsules shows similar total neutron yields with each hohlraum material; a direct indication that both $\mathrm{Au}$ and $\mathrm{Pb}$ produce comparable radiation drive in the integrated geometry required for ICF. The time of peak neutron emission, or "bang time", is measured and the neutron yield time
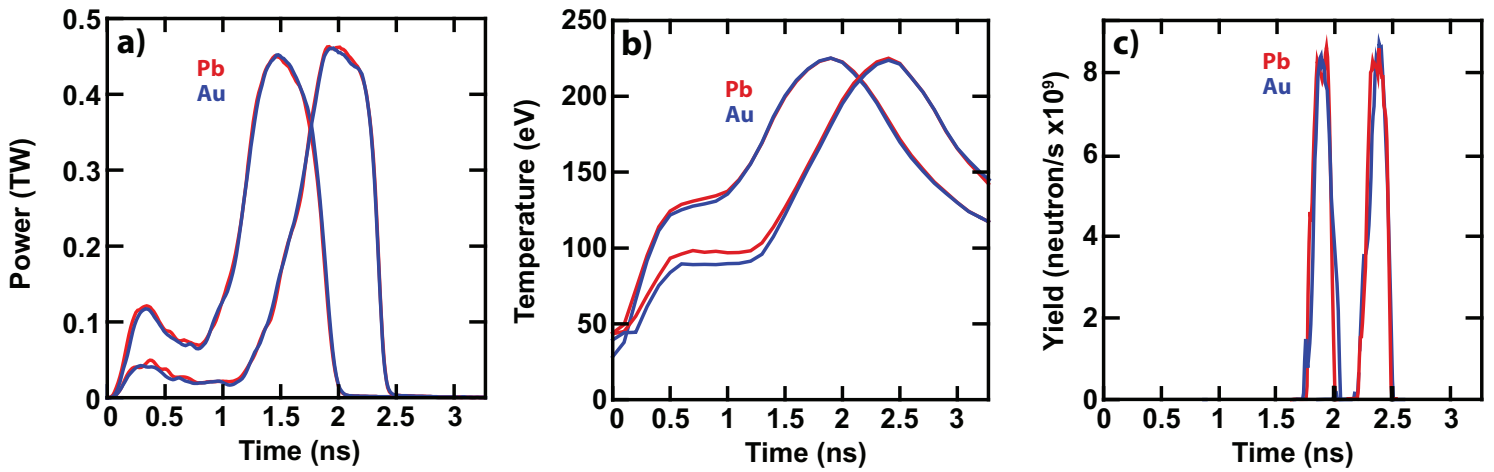

Figure $3 \mid$ (a) Total laser powers are shown for both pulse shapes for each target material. (b) The radiation temperature is measured with DANTE. (c) The neutron time history measured with the Neutron Temporal Diagnostic ${ }^{12}$ is compared for each hohlraum material and each pulse shape. 

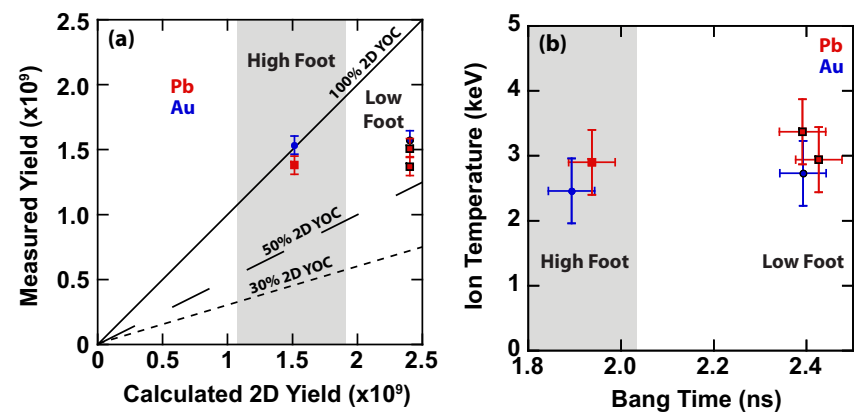

Figure $4 \mid$ (a) The measured neutron yield is plotted against the calculated 2D clean yield for lead (red squares) and gold (blue circles). The $2 \times$ foot pulse (no outline) and low foot pulse (black outline) show comparable yields. (b) The ion temperature is shown for a range of measured neutron bang times.

history is shown in Figure 3 (c). The $\mathrm{Au}$ and $\mathrm{Pb}$ hohlraums have very similar bang times for both pulse shapes, providing strong evidence that the capsule implosion velocities are similar and that the hohlraums produce a similar capsule drive environment. The total neutron yield is compared to simulations in Figure 4 (a). Twodimensional (2D) "YOC" contours of $100 \%, 50 \%$ and $30 \%$ are shown in Figure 4 (a), where the 2D YOC is defined as the ratio of the measured neutron yield to the predicted clean yield from $2 \mathrm{D}$ integrated (hohlraum + capsule) simulations in the absence of hydrodynamic instability and atomic mixing of capsule materials. The $\mathrm{Pb}$ and gold hohlraum performance for both the $2 \times$ foot and low-foot pulse shapes was similar: 2 D YOCs of $\sim 100 \%$ and $\sim 60 \%$ are found for the $2 \times$ foot and low foot pulse shapes, respectively. The simulated yields for the two pulse shapes dominate the variation in 2D YOC. The simulations predict relatively larger yield for the low foot pulse, which might be due to increased fuel convergence and uncertainties in the equation of state for $\mathrm{CH}$ that could affect shock timing. A $2 \mathrm{D}$ YOC of $\sim 40 \%$ is required for a successful ignition demonstration ${ }^{16}$, and we have met this threshold owing largely to the comparatively low fuel convergence.

The ion temperature and bang time are reported in Figure 4 (b). The measured ion temperatures range from 2.5 to $3.5 \mathrm{keV}$ (30-40 million degrees Kelvin), with the $\mathrm{Pb}$ targets having a slightly higher ion temperature for both pulse shapes. This increased ion temperature is consistent with the observed increase in radiation temperature in the foot of the pulse for the $\mathrm{Pb}$ hohlraum; simulations show a similar dependence. Ion temperatures approaching 50 million $\mathrm{K}$ are required for central hot spot ignition ${ }^{11}$, the mode of ignition proposed for IFE. At an ion temperature of 50 million $\mathrm{K}$ in the target core, the energy loses due to radiation and conduction are balanced by energy gain from fusion reactions according to calculations. The neutron bang times are also measured with the bang time variances dominated by the shot-to-shot variations in pulse shapes. The difference in bang time between $\mathrm{Au}$ and $\mathrm{Pb}$ is within the uncertainty of the measurement $(\sim \pm 50 \mathrm{ps})$.

In summary, we have shown that lead $(\mathrm{Pb})$ is a viable hohlraum material and performs as well as gold, providing essential data to discriminate between different predictive models. The measured hohlraum and capsule performance were characterized using a suite of diagnostics and show similar performance for lead and gold targets. For LIFE and cost-conscious future energy applications, lead is shown to be an appropriate target material.

\section{Methods}

The experiment in this work was performed at the Omega Laser facility at the Laboratory for Laser Energetics. A total of 38 laser beams enter the hohlraum through the pair of laser entrance holes. The laser beams are distributed on each side of the hohlraum and grouped into three beam cones defined by the angle the lasers enter the hohlraum relative to the symmetry axis. There are a total of 9 cone 1 beams entering the hohlraum at an angle of $21.4^{\circ}, 9$ cone 2 beams with an angle of $42.0^{\circ}$ and 20 cone 3 beams with an angle of $58.9^{\circ}$. Two lower beams (one cone 1 and one cone 2 ) that would pass through the diagnostic window have been disabled for this experiment, mildly breaking the up-down laser symmetry. Each laser beam delivers an average of $370 \mathrm{~J}$ of $351 \mathrm{~nm}$ laser light to the target, resulting in a total energy of $\sim 14 \mathrm{~kJ}$ on target. The laser beams are spatially smoothed using distributed phase plates and temporally smoothed using spectral smoothing by dispersion in 1D.

1. Dunne, M. et al. Timely Delivery of Laser Inertial Fusion Energy (LIFE). Fusion Sci. Technol. 60, 19-27 (2011).

2. Amendt, P., Dunne, M., Ho, D. D. \& Lindl, J. D. LIFE Pure Fusion Target Designs: Status and Prospects. Fusion Sci. Technol. 60, 49-53 (2011).

3. Callahan-Miller, D. A. \& Tabak, M. Progress in Target Physics and Design for Heavy Ion Fusion. Phys. Fluids 7, 2083-2091 (2000).

4. Jones, O. S. et al. Proof of Principle Experiments that Demonstrate Utility of Cocktail Hohlraums for Indirect Drive Ignition. Phys Plasmas 14, 056311 (2007)

5. Miles, R. et al. Challenges Surrounding the Injection and Arrival of Targets at LIFE Fusion Chamber Center. Fusion Sci. Technol. 60, 61 (2011)

6. Sources, J. M. et al. Role of the laboratory for laser energetics in the National Ignition Facility Project. Fusion Technol. 30, 492 (1996).

7. Moody, J. D. Multistep Redirection by Cross-Beam Power Transfer of UltrahighPower Lasers in a Plasma. 8, 344-349 (2012).

8. Glenzer, S. H. et al. Symmetric Inertial Confinement Fusion Implosions at UltraHigh Laser Energies. Science 327, 1228-1231 (2010).

9. Marshall, F. J. \& Bennett, G. R. A High-Energy X-ray Microscope for Inertial Confinement Fusion. Rev. Sci. Instrum. 70, 617-619 (1999).

10. Glenzer, S. H. et al. Demonstration of Ignition Radiation Temperatures in Indirect-Drive Inertial Confinement Fusion Hohlraums. Phys. Rev. Lett. 106, 085004 (2011).

11. Haan, S. W. et al. Point Design Targets, Specifications, and Requirements for the 2010 Ignition Campaign on the National Ignition Facility. Physics Plasmas 18, 051001 (2011).

12. Lerche, R. A., Phillion, D. W. \& Tietbohl, G. L., 25 ps Neutron Detector for Measuring ICF-Target Burn History. Rev. Sci. Instrum 66, 933 (1995).

13. Lide, D. R. ed. (2006-2007). CRC Handbook of Chemistry and Physics (87th ed.). pp. 14-13.

14. Collins, G. W. Measurements of the Equation of State of Deuterium at the Fluid Insulator-Metal Transition. Science 281, 1178-1181 (1998).

15. Dewald, E. et al. Dante Soft X-ray Power Diagnostic for the National Ignition Facility. Rev Sci Instrum 75, 3759-3761 (2004).

16. Chang, P. et al. Generalized Measurable Ignition Criterion for Inertial Confinement Fusion. Phys. Rev. Lett. 104, 135002 (2010).

\section{Acknowledgements}

We thank the staff of the OMEGA laser facility for their experimental support. This work was performed under the auspices of the U.S. Department of Energy by the Lawrence Livermore National Laboratory, under Contract No. DE-AC52-07NA27344. Additional support was provided by LLNL LDRD Grant No.11-ERD-075 and 11-SI-02.

\section{Author contributions}

J.S.R., P.A. and S.H.G. designed and prepared the experiment. Target fabrication and support was provided by A.N. and R.W. The data was analyzed by J.S.R. The paper was written by J.S.R., P.A., M.D. and S.H.G. Computational simulations were prepared by P.A. and D.M. Additional experimental and theoretical support was provided by L.J.A., J.D.L. and E.I.M.

\section{Additional information}

Supplementary information accompanies this paper at http://www.nature.com/ scientificreports

Competing financial interests: The authors declare no competing financial interests. License: This work is licensed under a Creative Commons

Attribution-NonCommercial-ShareALike 3.0 Unported License. To view a copy of this license, visit http://creativecommons.org/licenses/by-nc-sa/3.0/

How to cite this article: Ross, J.S. et al. Lead $(\mathrm{Pb})$ Hohlraum: Target for Inertial Fusion Energy. Sci. Rep. 3, 1453; DOI:10.1038/srep01453 (2013). 